\title{
Suraksh-Intrapartum Monitoring Application: In the Era of Distancing and Digitalization
}

\author{
Tanvi Desai ${ }^{1}$, Usha Kumari ${ }^{2}$, Suresh Vaidyanathan ${ }^{3}$
}

\begin{abstract}
This article introduces Suraksh, a mobile application that focuses on intensive intrapartum monitoring, shedding light on the novel concept of "distance monitoring", incorporating the pressing need of the hour for complete digitalization. It aims to reduce the burden on under-resourced set-ups, improving the turn-over time, ensuring timely intervention and referrals along with strengthening our clinical database for retrospective and observational studies. It targets paperless documentation and patient care in the advent of the "new normal" of minimal contact in health care set-ups. While this application is in its pilot phase, it has shown promising compliance and acceptance at our tertiary center, Sri Ramachandra Institute of Higher Education and Research, amongst senior obstetricians, postgraduates, interns and nursing staff. We welcome and promote adoption and wider usage of this application across all tiers of obstetric care.

Keywords: Digital partograph, Intrapartum monitoring, Intrapartum mortality, Mobile application, Suraksh.

Journal of South Asian Federation of Obstetrics and Gynaecology (2020): 10.5005/jp-journals-10006-1816
\end{abstract}

\section{BACKGROUND}

World over maternal mortality is unacceptably high. In 2017, over 295,000 women died during pregnancy and after childbirth. The vast majority (94\%) of these deaths occurred in low-resource settings and most could have been prevented.

The high number of maternal deaths in some areas of the world reflects inequalities in access to quality health services and highlights the stark wealth gap. In 2017, the maternal mortality rate in low income countries was 462 per 100,000 live births vs 11 per 100,000 live births in high income countries.

Intrapartum stillbirths and intrapartum-related neonatal deaths are a huge and potentially preventable burden, yet receive limited policy and programmatic attention. Innovative approaches are required to increase access to information that is vital to informed decision-making and improved care in settings where far too many babies do not cry at birth. ${ }^{2}$

In 2020, the COVID-19 pandemic has disrupted nations globally; forcing all disciplines to tune themselves to the "new normal" of hand hygiene and social distancing. Thus, in the field of obstetrics too, it is prudent that we modify our services for the safety of both the patient and obstetrician.

The battle with the primary issues highlighted above still continues; making development of technology-assisted tools - the need of the hour.

\section{TECHNIQUE}

Suraksh is a multiplatform labor monitoring application devised to monitor all phases of labor by inputting patient-specific data in a timely manner.

It is designed for smartphones (android and iOS), tablets, desktop computers (Windows and iOS) ${ }^{3}$ it works offline and gets synchronized when connected to the Internet.

The initial interface involves entering basic patient details when the antenatal mother is admitted to the labor ward. These details include patient identification and demographics: full name with an auto-capture of her photograph, hospital ID number, contact
1,2Department of Obstetrics and Gynecology, Sri Ramachandra Institute of Higher Education and Research, Chennai, Tamil Nadu, India ${ }^{3}$ Grafware International, Chennai, Tamil Nadu, India

Corresponding Author: Tanvi Desai, Department of Obstetrics and Gynecology, Sri Ramachandra Institute of Higher Education and Research, Chennai, Tamil Nadu, India, Phone: +91 9870481362, e-mail: tanvi.desai06@gmail.com

How to cite this article: Desai T, Kumari U, Vaidyanathan S. SurakshIntrapartum Monitoring Application: In the Era of Distancing and Digitalization. J South Asian Feder Obst Gynae 2020;12(5):271-276.

Source of support: Nil

Conflict of interest: None

number, birthdate, education, and husband's name along with date and time of admission (Fig. 1).

This is followed by preliminary obstetric data that include obstetric score, last menstrual period, corrected date of delivery, allergies, blood group, height, and weight. The software calculates expected date of delivery as well as body mass index from the inputted values.

Suraksh targets close monitoring of laboring patients with an attempt to reduce intrapartum morbidity and mortality; thus

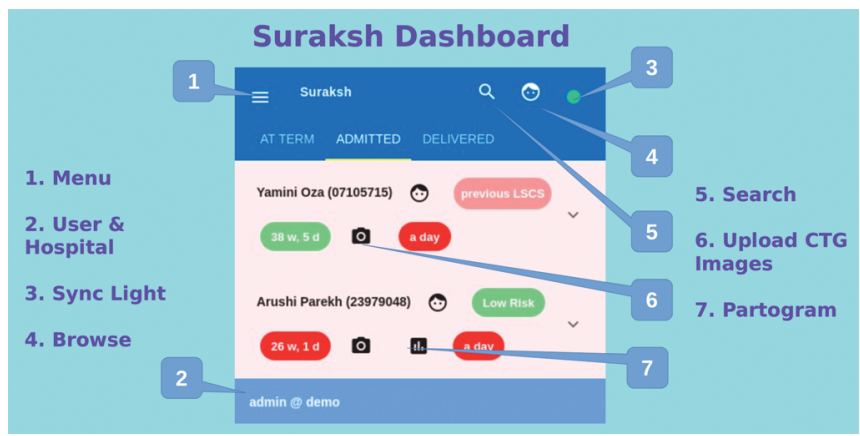

Fig. 1: Suraksh dashboard 


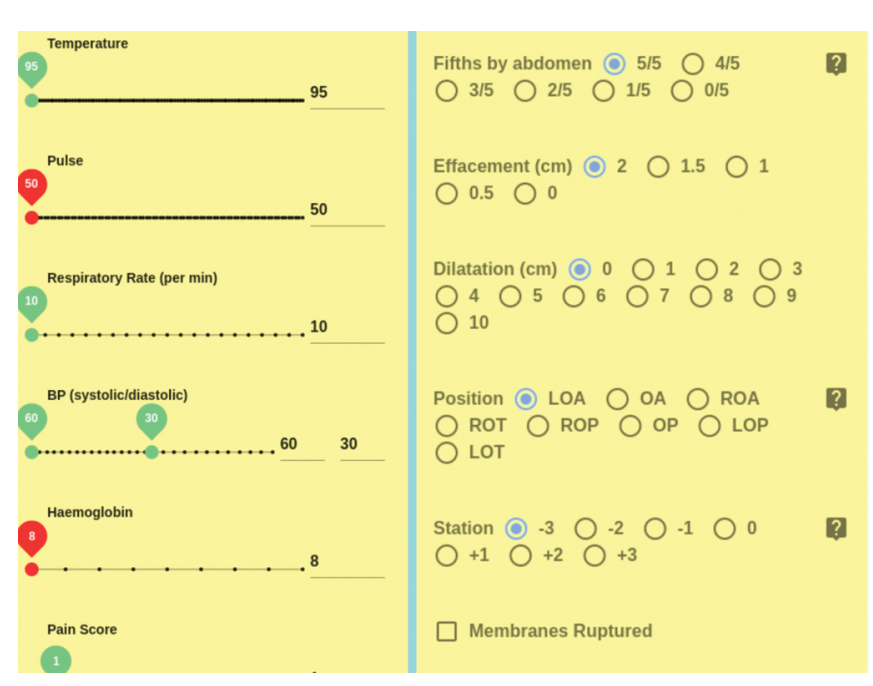

Fig. 2: Vital signs with pelvic examination

focusing on additional risk factors associated with each pregnancy. These include-though are not limited to-anemia, gestational hypertension, gestational diabetes mellitus, epilepsy, heart disease complicating pregnancy, fetal growth restriction, postdated pregnancy, etc.

The patients are divided into groups depending on the mode of delivery, that is, elective cesarean section or vaginal delivery (which may or may not be further classified into emergency cesarean section depending on the course of the labor) (Fig. 2).

Before the mother enters active labor, baseline examination findings are entered into her profile on the application which include blood pressure, pulse rate, respiratory rate, temperature, pain score, and a predelivery hemoglobin value which is of vital importance owing to the gross prevalence of anemia complicating pregnancies in India.

This is followed by detailed pelvic examination findings - fifths of the presenting part palpable per abdomen, effacement and dilation of the cervix as well as station and position of the presenting part (Fig. 3).

Further, the plan is inputted into the application if labor is spontaneous in onset or has been induced for the mother. The drugs administered, along with the time of administration, dosage, route, and type of drug (antibiotic, agents for induction/augmentation of labor), is punched in.

As the mother goes into active labor, the same can be activated on her profile following which number of contractions, duration of each contraction, rupture of amniotic membrane, fetal heart rate, and degree of molding can be recorded. The color of amniotic fluid is added as well as an auto-capture of the cardiotocograph of the fetal heart rate.

With each subsequent pelvic examination done to monitor labor, the same is updated on Surkash, which has a built-in software to simultaneously start plotting, the originally paperbased electronic partograph. This eliminates scope for delay in plotting a partograph; commonly seen in many labor and delivery setups. Surkash further recognizes any undue delays or deviations from normal labor, based on the partograph as well as the last done pelvic examination and sends out alert signals to all health care professionals registered on the application in the given institute. The presence of "help assistance" provides diagrammatic representation to assist with pelvic examination such as position of presenting part, suture lines, and station.

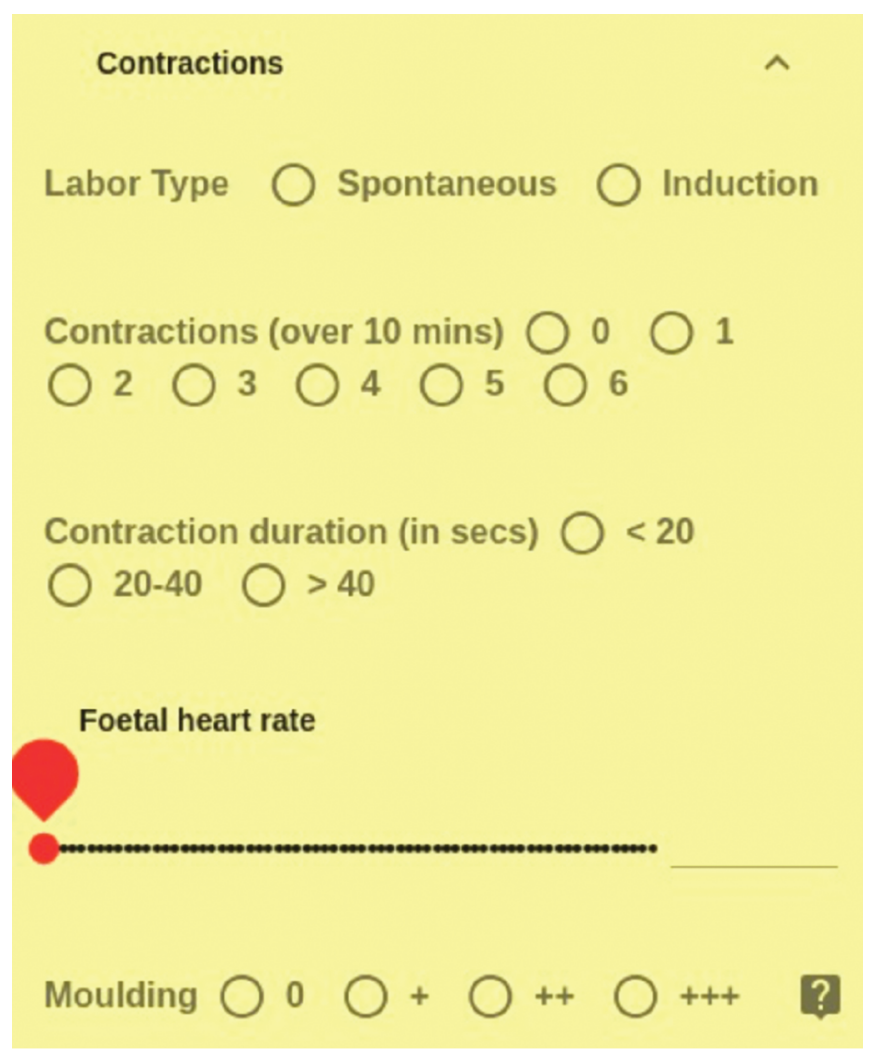

Fig. 3: Abdominal examination in active labor

This is followed by adding in delivery details such as mode of delivery, that is, assisted vaginal delivery (forceps and vacuum), normal vaginal delivery, emergency, or elective cesarean section; along with indication for the same. Details of the birth (date and time), APGAR score, weight, and sex of the newborn are documented. Any immediate complications encountered during the process of labor and delivery such as postpartum hemorrhage, stillbirth, and need for blood transfusions are registered in the application database.

The course of the mother in the hospital ends with a date and time of discharge along with an automated intrapartum report (PDF) generated by Suraksh, which has the course of labor along with the partograph and delivery details (associated complications included), which can be printed out if required or simply added to its internal database (Figs 4 to 6).

Suraksh not only assists the obstetrician and labor team with monitoring the patients' intrapartum findings but also includes data analytics for probabilistic modelling. The application generates a monthly report, in the form of a bar graph, shedding light on the total number of deliveries and percentage of vaginal vs cesarean vs assisted vaginal deliveries (Fig. 7). It uses artificial intelligence to correlate parameters and outcomes, such as the prevalence of various risk factors complicating the pregnancies and deliveries for the month such as anemia, epilepsy, cardiac disease, etc. (Fig. 8). Finally, Suraksh has deep learning algorithms to anticipate complications and gives us a comprehensive monthly report on the frequency of delayed intervention during the various deliveries, repeated need for the alert alarms; hence providing insight into shortcomings of the existing system and elucidating the need for further modification to improve maternal and fetal care in their outcomes. 


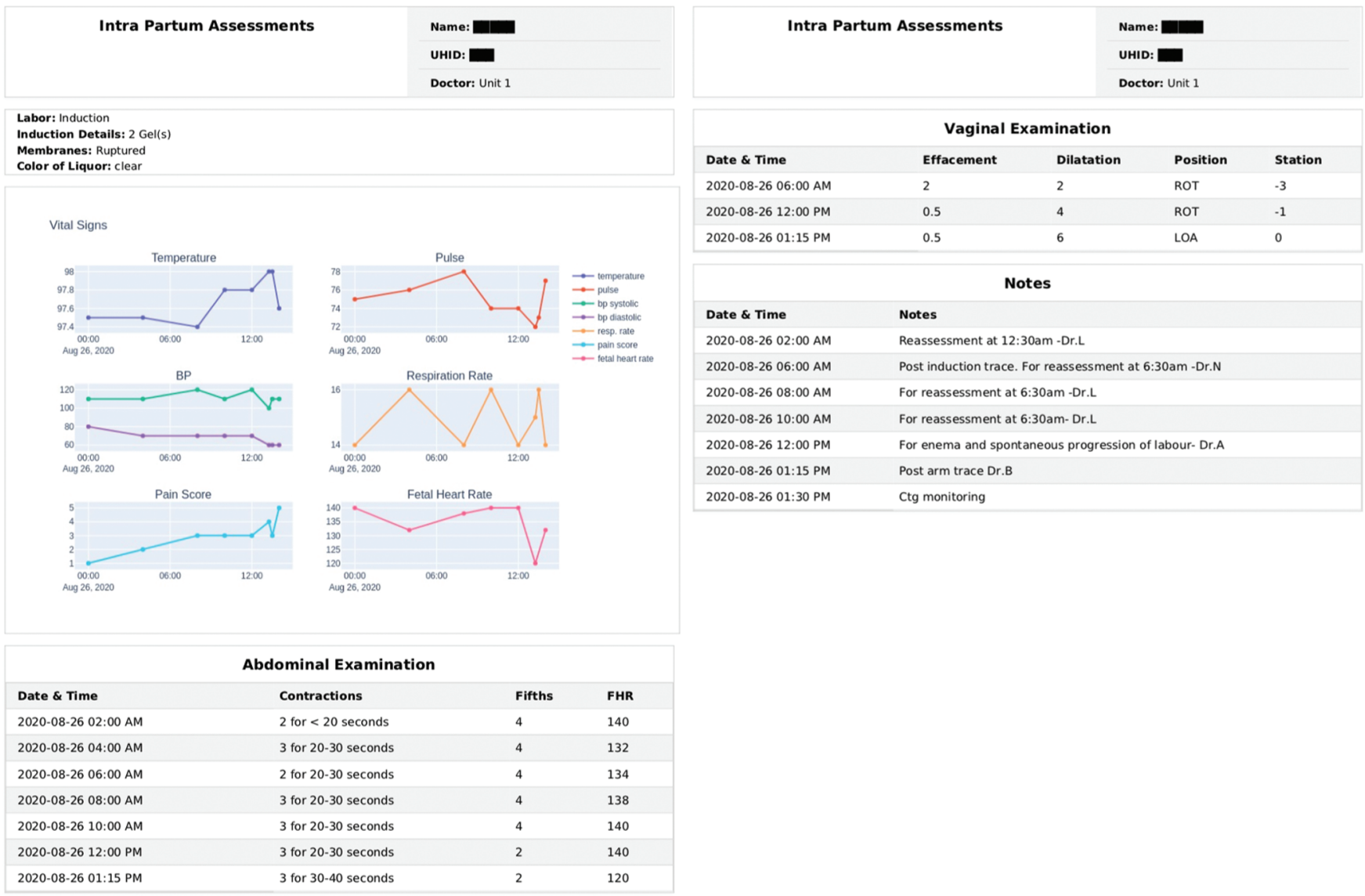

Fig. 4: Final intrapartum report

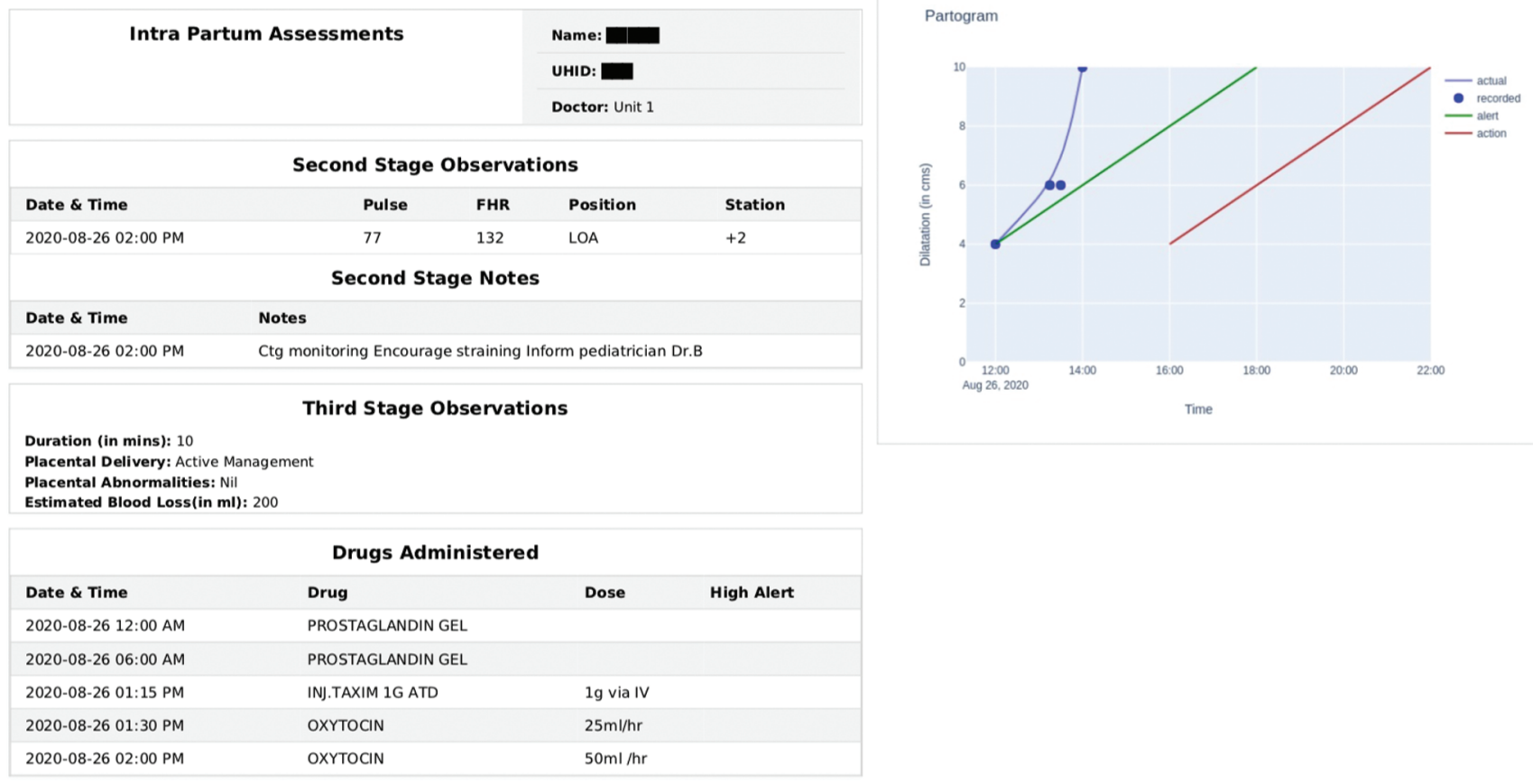

Fig. 5: Final intrapartum report 


\section{Parturition Details}

\section{Intra Partum Assessments}

\section{Name: \\ UHID:}

Doctor: Unit 1
Onset of Labor: Induction

Induction Details: 2 Gel(s)

Augmentation: ARM, Oxytocin

Delivery Mode: NVD

Delivery Time: 2020-08-26 08:38 AM

Gender: Girl

Weight (kg): 3.0

Apgar: 8, 9

Core blood taken: No

COVID-19 Status: Negative

\section{Singleton}

\section{Discharge Notes}

normal diet plenty of oral fluids dbf breast and perineal care $w / f$ increased bleeding $p / v$ svp for obs

\begin{tabular}{|lllll|}
\hline & \multicolumn{3}{c}{ Post Delivery Assessment } \\
\hline Time & Pulse & BP & Uterus & Vaginal Bleeding \\
\hline 15 minutes & 74 & $110 / 80$ & Contracted & Normal \\
\hline 30 minutes & 74 & $110 / 70$ & Contracted & Normal \\
\hline 1 hour & 80 & $120 / 80$ & Contracted & Normal \\
\hline On Discharge & 86 & $110 / 70$ & Contracted & Normal \\
\hline
\end{tabular}

\section{Discharge Check List}

Urine voided:

\begin{tabular}{lll} 
Item & Count & Checked \\
\hline Pads & 14 & \\
\hline Suture/Needles & 2 &
\end{tabular}

Conducted By: $D_{m} m$

Assisted By: DmB

Staff Nurse: Mss

Date/Time: 2020-08-26 10:30 AM

- Suraksh, v1.64

Generated 2020-09-02 16:13

C2019,2020

Fig. 6: Final intrapartum report 

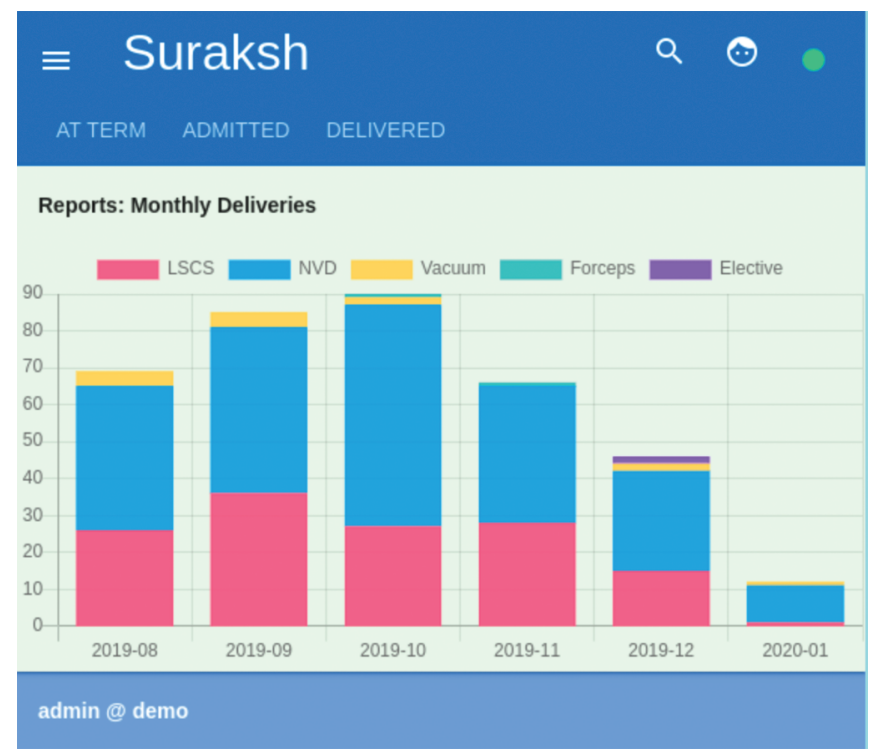

Fig. 7: Monthly analysis of type of deliveries

The alarms and escalation settings can further be modified and customized based on the kind of setup as well as personal convenience-ranging from time of admission of the patient to entering preliminary labor data to time fixed for successive evaluation of progress of the patient in active labor. This can vary from across setups, depending on the patient load and the number of members available on the labor and delivery team.

With regard to data storage and security, all data are encrypted during transmission with the same technology used by bank and credit card sites. Each hospital has its own distinct database and no data is shared. Server data are used only for syncing and are not visible to any third party.

\section{Discussion}

Suraksh was introduced to the Department of Obstetrics and Gynecology at SRIHER in October 2019 and we have had the experience of monitoring 600 deliveries till August 2020. An initial debriefing was given to the labor and delivery team, regarding the usage and features of the application. The team included consultants, postgraduates, interns, and nursing staff. In the era of digitalization, that we all now belong to, compliance and uptake of a new method of intrapartum monitoring were unchallenging.

The partograph, a paper tool for recording clinical data during childbirth, has demonstrated a reduction in obstructed labor when used to track patients. ${ }^{4}$ It has been endorsed by the World Health Organization (WHO) for highlighting deviations from normal labor, especially prolonged labor. Despite being a very helpful tool, usage of the partograph has proven to be a barrier in itself. Many setups have admitted to plotting the partograph only after delivery for documentation purposes. Lack of knowledge or plotting by underskilled workers can lead to misinterpretation of progress of labor and untimely intervention. Surkash attempts to overcome these technical difficulties by digitalizing the partograph. This reduces the scope of human error, delays in plotting the course of labor, and minimizes the chances of the error in judgment.

Introduction of any innovation requires the new design to have a balance between sophistication and user-friendliness. Suraksh
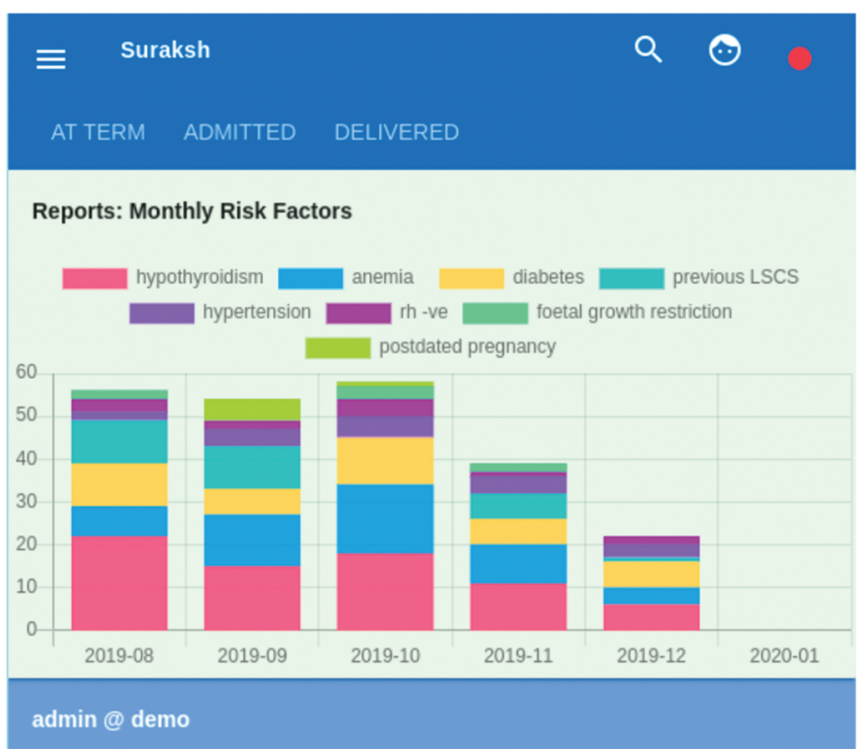

Fig. 8: Monthly analysis of risk factors associated with pregnancies

has attempted to achieve this with the designing of every feature of the application.

While it is agreed that an electronic application could never replace clinical skill and judgment, which comes from years of experience and experimental learning, Surkash has been deployed not as a replacement, but as a supplement to organize and direct less experienced individuals to provide optimum care under the directives of senior obstetricians who are monitoring labor through the application.

Despite adding structure to labor ward protocols, Surkash does not reduce the flexibility of the process of intrapartum monitoring. Alert signs and signals as well as frequency of examination required can be customized for each patient depending on the case scenario.

To enhance the ease of use, it was ensured that the application was available on desktop computers in our labor wards, such that individual log-in through mobile phones was not required every time to input data. However, accountability was not compromised, as each update needed a counter sign of the name of the concerned professional.

\section{Clinical Significance}

The latest available data suggest that in the most high-income and upper middle income countries, more than $90 \%$ of all births benefit from the presence of a trained midwife, doctor, or nurse. However, fewer than half of all births in several low-income and lower middle income countries are assisted by such skilled health personnel. ${ }^{5}$

In 2015, there were 25.121 million live births in India and 1.201 million under-5 deaths (under-5 mortality rate 47.81 per 1,000 live births). About 0.696 million (57.9\%) of these deaths occurred in neonates. There were disparities in child mortality across states [from 9.7 deaths (Goa) to 73.1 deaths (Assam) per 1,000 live births] and regions [from 29.7 deaths (the south) to 63.8 deaths (the northeast) per 1,000 live births]. Overall, the leading causes of under- 5 deaths were preterm birth complications ( $27.5 \%$ of under- 5 deaths), pneumonia (15.9\%), and intrapartum-related events (0.139 million; $11.6 \%$ ), with cause-of-death distributions varying across states and regions. ${ }^{6}$ 
Three million babies are stillborn each year and 3.6 million die in the first month of life. In India, early neonatal deaths make up fourfifths of neonatal deaths and infant mortality and three-quarters of under-5 mortality. ${ }^{7}$

In a study done by Bapat et al., of 105 stillbirths studied in slum settlements in India, 65 were fresh (62\%) and obstetric complications dominated the cause classification. Of 116 neonatal deaths, 87 were early and the major causes were intrapartumrelated $(28 \%)$, prematurity $(23 \%)$, and severe infection $(22 \%)^{7}$

The main factors that prevent women from receiving or seeking care during pregnancy and childbirth are poverty, distance to facilities, lack of information, inadequate and poor quality services, and cultural beliefs and practices.

To improve maternal health, barriers that limit access to quality maternal health services must be identified and addressed at both health system and societal levels.

As defined in the Ending Preventable Maternal Mortality Strategy, ${ }^{8}$ WHO is working with partners in supporting countries toward:

- Addressing inequalities in access to and quality of reproductive, maternal, and newborn health care services;

- Ensuring universal health coverage for comprehensive reproductive, maternal, and newborn health care;

- Addressing all causes of maternal mortality, reproductive and maternal morbidities, and related disabilities;

- Strengthening health systems to collect high quality data to respond to the needs and priorities of women and girls; and

- Ensuring accountability to improve quality of care and equity.

Suraksh helps us to address these unresolved issues by incorporating close monitoring by skilled health care workers, with a viable option of distance electronic monitoring along with paperless patient care, without compromising the quality of health services.

The finer settings of the application can be customized such that it can be used through the entire spectrum of health care in a country such as India.

Primary health care centers struggle with the availability of skilled obstetric services. The user-friendliness of Surkash, especially with the presence of graphics along with text, enable nursing staff, general practitioners, and even interns to input data. The progress can be monitored by a more skilled obstetrician with a base far away from the primary health center, thus overcoming the obstacle of distance.

Secondary health centers such as taluk and district hospitals are equipped with skilled personnel; however, these centers may not be armed with the equipment needed to undertake high-risk obstetrics. In such cases, with close monitoring by experienced obstetricians through Suraksh, timely intervention and decisionmaking for referral to a higher center can be truly rewarding, thus reducing maternal and perinatal morbidity and mortality.

Organized record-keeping and data storage has always been a struggle for populous and understaffed countries such as India.
Tertiary healthcare centers, corporate hospitals in metropolitan cities, and academic institutes such as ours (SRIHER) can now have an extensive database which has systematic records stored. Data can be used for research purposes, predictive analysis and to bring about modifications to improve the standard and quality of maternal and fetal care.

In academic and teaching institutes, postgraduates and interns can have a complete hands-on experience with labor and delivery processes without risking patient care due to the option of distance monitoring by senior consultants with the aid of Suraksh.

\section{Conclusion}

Innovation always comes with initial teething problems; the acceptance and compliance curve can be steep. While we have had promising preliminary results on both fronts of acceptance and compliance at our institute, we recognize that this is observation based out of a single department at a single tertiary care center. Further large-scale adoption of Suraksh is needed for it to stand the test of time. That being said, we believe digitalization along with distance monitoring is the route for ironing out the disparity in obstetric services in a populous country such as India, where the ratio of patient to healthcare workers and reach of services nationwide is always going to be a Herculean struggle.

\section{Ethical Approval}

This study does not contain any studies with human participants or animals performed by any of the authors.

\section{References}

1. Trends in maternal mortality: 2000 to 2017: estimates by WHO, UNICEF, UNFPA, World Bank Group and the United Nations Population Division. Geneva: World Health Organization; 2019.

2. Lawn J, Shibuya K, Stein C. No cry at birth: global estimates of intrapartum stillbirths and intrapartum-related neonatal deaths. Bull World Health Organ 2005;83(6):409-417.

3. https://vplan.in/suraksh/.

4. WHO, Maternal Health and Safe Motherhood Programme. 1994. Preventing Prolonged labour: a practical guide: the partograph. Accessed online 1 February 2015: http://apps.who.int/iris/ handle/10665/58903.

5. World Health Organization and United Nations Children's Fund. WHO/UNICEF joint database on SDG 3.1.2 Skilled Attendance at Birth. Available at: https://unstats.un.org/sdgs/indicators/database/.

6. National, regional, and state-level all-cause and cause-specific under-5 mortality in India in 2000-15: a systematic analysis with implications for the Sustainable Development Goals Crossref DOI link: https://doi.org/10.1016/S2214-109X(19)30080-4 Published: 2019-06.

7. Bapat U, Alcock G, More NS, et al. Stillbirths and newborn deaths in slum settlements in Mumbai, India: a prospective verbal autopsy study. BMC Pregnancy Childbirth 2012;12:39. DOI: 10.1186/1471-239312-39.

8. Strategies towards ending preventable maternal mortality (EPMM). Geneva: World Health Organization; 2015. 